\title{
NEURODIDÁCTICA Y FORMACIÓN CONTINUADA DE DOCENTES PARA LA ATENCIÓN A LA DIVERSIDAD EN COLOMBIA
}

\author{
Neurodidactics and continuous training of teachers for attention to diversity in Colombia
}

\section{Luz Mabel Gonzalez Altahona ${ }^{1}$ \\ Timarly Melo Meza ${ }^{2}$ Adriana Pineda Robayo ${ }^{3}$}

RESUMEN: La atención a la diversidad en Colombia es considerada un campo fundamental de cualificación de la acción pedagógica de los docentes que desempeñan su labor en los grados transición y primero de Básica Primaria. El articulo presenta los resultados de una investigación desarrollada en el contexto de la Maestría en Neuropedagogia y el objetivo es evidenciar el aporte de la Neuro didáctica a la acción pedagógica de los docentes de educación infantil, especialmente en el tema de la atención a la diversidad. El estudio se desarrolla a partir de los aportes teóricos de Campos (2010); Giroux (1997); Artavia y Fallas (2012). Se trata de un estudio cualitativo que, desde la Investigación Acción, busca generar espacios de reflexión y transformación de la acción pedagógica del docente, alrededor de la atención a la diversidad. Los resultados evidencian que la estrategia diseñada a partir de los parámetros de la neurociencia cognitiva y aplicada en cinco Instituciones Educativas del municipio de Puerto Colombia (Atlántico), Colombia, tiene una incidencia positiva en la práctica del docente como escenario de deconstrucción y reconstrucción de los parámetros que guían su quehacer, así como de posibilidad de reconfiguración de una práctica pertinente, significativa y respetuosa de la diversidad, los intereses, necesidades y realidades de los estudiantes.

Palabras Clave: Neuro didáctica, Atención a la Diversidad, Acción Pedagógica del Docente.

\footnotetext{
${ }^{1}$ Luz Mabel Gonzalez Altahona. Magister en Neuropedagogia. Universidad del Atlántico. Email: luzma_1a@yahoo.es. Código Orcid: https://orcid.org/0000-0002-0171-6583.

2 Timarly Melo Meza. Magister en Neuropedagogia. Universidad del Atlántico. Email: timysmm@hotmail.com. Código Orcid: https:/orcid.org/0000-0002-8811-0882.

${ }^{3}$ Adriana Pineda Robayo. Doctora en Educación: Universidad del Atlántico.

Email: adripinedarobayo@gmail.com. Código Orcid: https://orcid.org/0000-0001-5990-6833
} 
ABSTRACT: Attention to diversity in Colombia is considered a fundamental field of qualification of the pedagogical action of teachers who carry out their work in the transitional and first grades of Basic Primary. The article presents the results of an investigation developed in the context of the Master's Degree in Neuropedagogy and the objective is to demonstrate the contribution of Neuro-didactics to the pedagogical action of early childhood education teachers, especially on the subject of attention to diversity. The study is developed from the theoretical contributions of Campos (2010); Giroux (1997); Artavia \& Fallas (2012). It is a qualitative study that, from Action Research, seeks to generate spaces for reflection and transformation of the pedagogical action of the teacher, around the attention to diversity. The results show that the strategy designed from the parameters of cognitive neuroscience and applied in five Educational Institutions of the municipality of Puerto Colombia (Atlántico), Colombia, has a positive impact on teacher practice as a scenario for deconstruction and reconstruction of the parameters that guide their work, as well as the possibility of reconfiguring a pertinent, meaningful, and respectful practice of the diversity, interests, needs and realities of students.

Key Words: Neuro-didactics, Attention to diversity, the pedagogical action of early childhood education teachers

RESUMO: A atenção à diversidade na Colômbia é considerada um campo fundamental de qualificação da ação pedagógica dos professores que realizam seu trabalho nas séries transitórias e primeiras do Ensino Fundamental. O artigo apresenta os resultados de uma pesquisa realizada no âmbito do Mestrado em Neuropedagogia e tem como objetivo demonstrar a contribuição da Neurodidática para a ação pedagógica de professores de educação infantil, especialmente na área da atenção à diversidade. O estudo é desenvolvido a partir das contribuições teóricas de Campos (2010); Giroux (1997); Artavia e Fallas (2012). É um estudo qualitativo que, a partir da Pesquisa-Ação, busca gerar espaços de reflexão e transformação da ação pedagógica do professor, em torno da atenção à diversidade. Os resultados mostram que a estratégia desenhada a partir dos parâmetros da neurociência cognitiva e aplicada em cinco Instituições de Ensino do município de Puerto Colombia (Atlántico), Colômbia, tem um impacto positivo na prática docente como cenário para a desconstrução e reconstrução do parâmetros que norteiam o seu trabalho, bem como a possibilidade de reconfigurar uma prática relevante, significativa e respeitosa da diversidade, interesses, necessidades e realidades dos alunos.

Palavras-chave: Neurodidática , Atenção à diversidade, Ação pedagógica dos professores 


\section{INTRODUCCIÓN}

La educación como ciencia social, compuesta por complejos elementos y actores que interactúan constantemente, en las últimas décadas ha experimentado cambios importantes en cuanto a las perspectivas que la orientan. Producto de esta dinámica es posible verificar la solución de algunas tensiones y el surgimiento de otras. Un ejemplo de esto es el tema de la atención a la diversidad y sus implicaciones en un contexto que le exige a la escuela y específicamente al profesor la garantía de preservación de todos los derechos de la infancia y el despliegue de un quehacer orientado a favorecer su desarrollo integral.

El artículo presenta los resultados de una investigación desarrollada en el contexto de la Maestría en Neuro pedagogía de la Universidad del Atlántico y se basa en la convicción de la capacidad que poseen los docentes para reconfigurar su quehacer pedagógico hacía la pertinencia en lo que respecta a la atención a la diversidad presente en sus estudiantes, a la luz de los planteamientos de la neurociencia cognitiva y específicamente de la neurodidáctica.

Lo anterior implica el reconocimiento de nuevos significados y escenarios educativos, coherentes con la individualidad, ritmo, interés, necesidad, potencialidad, capacidad y estilo de aprendizajes de cada estudiante.

En este sentido, una de las más grandes preocupaciones de la educación actualmente es la calidad del servicio enmarcado en principios de equidad e inclusión como escenarios propicios para el desarrollo integral del sujeto. A partir de este concepto surge la necesidad de contextualizar un currículo coherente con la diversidad y articulado a prácticas pedagógicas innovadoras que encuentren en la neurodidáctica el camino para potenciar las habilidades y capacidades de los estudiantes.

La diversidad se reconoce como un principio orientador, fundamental en la acción pedagógica del profesor, por su capacidad para favorecer la trayectoria de las niñas, niños, adolescentes y jóvenes en el sistema educativo, asegurando su ingreso, permanencia, promoción y egreso en el contexto del reconocimiento de una educación para todos con igualdad de oportunidades, y atenta a las características, posibilidades y expectativas de cada uno.

Dicha educación se enmarca en la promoción del desarrollo, aprendizaje y participación, con pares de su misma edad, en un ambiente de aprendizaje común, sin discriminación o exclusión alguna, 
y que garantiza los derechos, apoyos y ajustes razonables requeridos en un proceso educativo capaz de superar las barreras existentes

\section{NEUROCIENCIA, EDUCACIÓN Y ACCIÓN PEDAGÓGICA DEL DOCENTE}

La Neuroeducación como interdisciplina y transdisciplina que promueve la integración de las ciencias de la educación con aquellas que se ocupan del desarrollo neurocognitivo de la persona humana, de acuerdo con Battro y Cardinali (1996), integra conceptos teóricos del funcionamiento cerebral a nivel cognitivo para ser aterrizados en la práctica educativa de manera innovadora y con gran impacto en su finalidad de potenciar el desarrollo cerebral humano.

Los resultados de las investigaciones en Neuroeducación han contribuido al entendimiento del aprendizaje humano desde diferentes perspectivas, por lo tanto, es importante que los docentes conozcan, se empoderen y logren implementar en sus aulas los aportes de la neurociencia cognitiva al campo educativo, asociados a la valoración del potencial que tiene el cerebro infantil para desarrollarse y aprender si se favorecen los ambientes y oportunidades adecuadas.

Sin embargo, de acuerdo con Campos (2010), aunque persiste la brecha entre educación y neurociencias, reflejada en el alejamiento entre lo que saben los educadores acerca del desarrollo cerebral infantil y lo que hacen en su práctica pedagógica, es posible superarla a partir del conocimiento de las bases neurales del aprendizaje y las funciones del cerebro relacionadas con la emoción, la memoria, el lenguaje, los sistemas sensoriales y motores, la atención los comportamientos como aspectos esenciales en el salón de clase.

El estudio plantea la necesidad de generar espacios de reflexión acerca de la acción pedagógica de los docentes de Educación Infantil, en el objetivo de trascender de un esquema educativo tradicional y homogeneizante, hacia una propuesta que considere el aporte fundamental de la neurociencia cognitiva a la pedagogía.

En este escenario, la Neuro pedagogía garantiza la consolidación de nuevas interpretaciones y comprensiones, producto de la sinergia transdisciplinar entre esto dos campos, desde la cual es posible observar el quehacer del profesor, la enseñanza, el aprendizaje, el currículo y la relación maestro, estudiante, ambiente, sociedad y cultura.

Es decir, una nueva forma de hacer, pensar y entender la educación y su relación con el contexto social y cultural de los actores que intervienen en el proceso. 
Lo anterior es ratificado por Peralta (2005), quien menciona que observar el fenómeno educativo a la luz de los aportes de la neurociencia, garantiza la transformación del concepto de aprendizaje como expresión externa, hacia una interpretación fundamentada en las bases neurobiológicas de los procesos mentales que se movilizan cuando se aprende, y la importancia de que esta movilización este transversalizada por la emoción y la experiencia de acercamiento lúdico y placentero al conocimiento.

Actualmente, la sinergia entre educación y neurociencias ratifica la importancia de comprender y valorar la diversidad presente en los niños que se adaptan, aprenden, se relacionan en un contexto que consciente o inconscientemente aprecia más la homogeneización que la diferencia. Es así como la importancia de la acción pedagógica del docente y su capacidad movilizadora de estrategias pertinentes y conectadas con la formación integral del estudiante es proporcional como lo mencionan Pineda y Felicetti (2018, p. 408) al "fortalecimiento de los saberes disciplinar y didáctico del profesor y a los procesos reflexivos sobre su quehacer en el aula".

Igualmente, Giroux (1997) plantea la importancia de que el docente cuente con los referentes teóricos y metodológicos necesarios para comprender, valorar y trabajar desde las formas en que se construye la diferencia, sus representaciones y prácticas.

En este sentido, cuando los niños inician su tránsito por el contexto educativo en el grado transición y primero de la Básica primaria (entre los 5 y 6 años), son caracterizados por los docentes como sujetos altamente creativos, imaginativos y con altos niveles de neuro plasticidad, pero también como poseedores de competencias y habilidades desarrolladas a partir de las interacciones que establece con su contexto familiar, cultural y social, es decir el docente reconoce el carácter diverso y único del niño al ingresar al sistema educativo.

\section{ATENCION A LA DIVERSIDAD}

Cuando se habla de diversidad necesariamente se hace alusión a las diferencias y características individuales propias del ser humano, que le otorgan el carácter de único, a la que vez nutren y enriquecen el proceso de intercambio de subjetividades, la interacción y retroalimentación, fundamentales en la construcción de su razón.

El concepto de diversidad ha tenido múltiples acercamientos y formas de representación, como lo mencionan Manosalva y Tapia (2009 p.91), al afirmar que "la diversidad como toda cuestión 
sociohistórica, política y cultural ha mostrado múltiples y distintos modos de representarla y comprenderla".

Específicamente, en el contexto educativo, el concepto de Diversidad va más allá del acceso, situándose como argumento desde el cual se preserva el derecho de todos los actores a llevar una vida digna, reconociendo y valorando las diferencias personales como eje transversal de los valores que, aprendidos desde la vivencia y la experiencia, garantizan la construcción de una sociedad democrática, plural y tolerante.

En este sentido, Artavia y Fallas (2012, p. 48) ratifican que diversidad no es sinónimo de diferencias, por el contrario, se enriquece con estas, siempre y cuando sea asumida como "un proceso dinámico capaz de responder a diferentes ámbitos del ser humano; a grupos culturales o étnicos; a género, clase social, estilos y formas particulares de aprender; a personas con discapacidad o sobre capacitadas, entre otras características personales y sociales.

La diversidad entendida como consecuencia directa de las diferencias y como cualidad inherente, connatural al ser humano, implica también la conciencia frente al hecho de que la organización de las Instituciones Educativas debe contemplar las estrategias necesarias para gestionarla y convertirla en un estilo pedagógico que adquiere vida en el quehacer del docente en el aula.

En este sentido, se ratifica la importancia de que el profesor en su quehacer logre dar testimonio de una visión amplia y pertinente del concepto de diversidad, teniendo en cuenta que esta perspectiva le facilita el establecimiento de sinergias efectivas, que, desde el respeto, reconocimiento y valoración de la diferencia, es capaz de producir nuevas y enriquecidas maneras de ser y estar en el mundo.

Así, desde la investigación se diseño una propuesta de formación dirigida a los docentes y conectada con la posibilidad de enriquecer los referentes que orientan su acción pedagógica en torno a como atender de manera pertinente y significativa a los niños y niñas. Partiendo de la premisa de que el cerebro es el órgano más complejo del cuerpo, pues rige toda la actividad y marca los patrones de comportamiento; además, su complejidad hace que sea único e irrepetible, y por consiguiente que cada mente sea un universo en sí misma. Esto implica que cualquier experiencia y todo proceso educativo influyan o puedan influir de manera distinta en cada persona, según sea su cerebro, haciendo que cada uno sea un ser diferente, único y singular.

Cuando se habla de este término dentro del ámbito educativo, se entiende que las diferencias entre los estudiantes son las características individuales propias de cada uno de ellos que hacen que sean únicos. 
El concepto de Diversidad, indica que cada estudiante tiene unas necesidades educativas individuales, propias y específicas a la hora de acceder a sus procesos de aprendizaje, por lo tanto, la educación está llamada a evolucionar y para dar respuesta a estas necesidades. Desde su aparición, la educación especial se ha ido transformando y modificando según los atributos que se han otorgado a la educación en general, relacionada, a su vez con marcos políticos, ideológicos, sociales y económicos más amplios. Si bien en la edad antigua, estaba bien aceptado el infanticidio ante la presencia de anormalidades en los niños, más tarde, concretamente hasta el Siglo XVII, se rechazaba y repudiaba a las personas con estas características.

Birch en el año (1974), citado por Bautista (1993), define la Diversidad como un proceso que pretende unificar las educaciones ordinaria y especial con el objetivo de ofrecer un conjunto de servicios a todos los niños, en base a sus necesidades de aprendizaje” (Bautista, 1993, p. 31), la define como

"la integración temporal, instructiva y social de un grupo de seleccionado de niños excepcionales, con sus compañeros normales, basada en una planificación educativa y un proceso 'programador evolutivo e individualmente determinado. Esta integración requería una clasificación de responsabilidades entre el personal educativo regular y especial y el personal administrativo, instructor y auxiliar".

Más adelante, en el año 1996, Tony Booth la define como un proceso consistente en responder a la diversidad de necesidades de todos los alumnos y satisfacerlas mediante una mayor participación en el aprendizaje, las culturas y las comunidades, así como en reducir la exclusión dentro de la educación y a partir de ella. Siguiendo en la línea del tiempo, en el año 2008, la UNESCO menciona la inclusión como:

"un proceso de abordaje y respuesta a la diversidad de las necesidades de todos los alumnos a través de la creciente participación en el aprendizaje, las culturas y las comunidades, y de la reducción de la exclusión dentro y desde la educación" (p.11).

Según esa misma Conferencia Internacional de Educación de 2008, el concepto de inclusión ha evolucionado hacia la idea que todos los niños, las niñas y los jóvenes deberían tener condiciones y oportunidades equivalentes de aprendizaje en diferentes tipos de escuelas independientemente de sus antecedentes sociales y culturales, así como de sus diferencias en las habilidades y capacidades. 
Para Borsani y Gallicchio (2009, p. 33), la Diversidad se puede integrar a partir del momento en que se estima que el niño se encuentra en condiciones de ser sujeto activo del proceso de aprendizaje sistemático y de alcanzar los objetivos escolares propuestos, intentarlo prematuramente o a destiempo puede condenarlo al fracaso. De acuerdo con los anteriores planteamientos es posible señalar que el concepto de diversidad ha tenido múltiples acercamientos y formas de representación. Tal como lo mencionan Manosalva y Tapia (2009), "La diversidad como toda cuestión sociohistórica, política y cultural ha mostrado diversas caras, implicando distintos modos de representarla y comprenderla" (p.91).

Frente a esta postura, Artavia y Fallas (2012) aseguran que: El término diversidad no es sinónimo de diferencias, por el contrario, se enriquece con estas. Se entiende como un proceso dinámico que responde a diferentes ámbitos del ser humano; a grupos culturales o étnicos; a género, clase social, estilos y formas particulares de aprender; a personas con discapacidad o sobre capacitadas, entre otras características personales y sociales. (p. 48).

De igual manera Colombia no se queda atrás en la construcción de Lineamientos de Política para Educación Inclusiva que tenga como punto de partida el reconocimiento de la diversidad, el valor y el respeto por la diferencia, promoviendo la articulación de estrategias y acciones de fomento que, en el marco de la autonomía universitaria, promuevan el acceso, la permanencia y la graduación de estudiantes pertenecientes a los diferentes grupos poblacionales.

En el Plan Decenal de Educación 1996-2005, en su estrategia "promoción de la equidad en el sistema educativo", reconoce que somos un país multiétnico y pluricultural con la necesidad de responder a esa diversidad.

En este mismo sentido, el plan sectorial del MEN - Plan Sectorial 2010-2014, en el documento N. 9, plantea: (...) la política educativa se estructura alrededor de una premisa fundamental: una educación de calidad es aquella que forma ciudadanos con valores éticos, respetuosos de lo público, que ejercen los derechos humanos, cumplen sus deberes sociales y conviven en paz. Una educación que genera oportunidades legítimas de progreso y prosperidad, competitiva y que contribuye a cerrar las brechas de inequidad. Una educación centrada en la institución educativa, que compromete la participación de toda la sociedad en un contexto diverso, multiétnico y pluricultural.

En el año 2017 el Ministerio de Educación Nacional emite el Decreto 1421, con el que se reglamenta en el marco de la educación inclusiva la atención educativa a la población con discapacidad, con el fin de cerrar las brechas de desigualdad y para garantizar una educación de calidad en 
condiciones de equidad en donde uno de sus principios es "El respeto por la diferencia y la aceptación de las personas con discapacidad como parte de la diversidad humana”.

Siendo así, cabe resaltar que el nivel máximo de las posibilidades de cada niño, joven y adulto es diverso, como diferente es su desarrollo y diversa es la personalidad, las aptitudes, y la capacidad mental y física en cada uno, es de esta forma que el derecho a la educación en la diversidad es jurídicamente reconocido como tratado internacional.

La gestión de la diversidad es, por tanto, una preocupación de la mayoría de los equipos directivos que se enfrentan cada día a escuelas, con el propósito de orientar una adecuada y coherente progresión de oportunidades educativas, proponiendo un conjunto de experiencias de aprendizaje que buscarán fortalecer el trabajo pedagógico.

El derecho a la Diversidad, en una institución educativa, va más allá del acceso, pues debe dar respuesta a todos y no atender a unos en detrimento de otros, en el derecho de todos los estudiantes a llevar una vida digna. Se trata de entender la diversidad como soporte de una serie de valores de importancia capital para la construcción de una sociedad democrática, plural y tolerante. Educar en la diversidad es reconocer las diferencias existentes entre las personas.

Todos estos cambios han sido gracias a los aportes que ha hecho la Neurociencia en cuanto al cambio de paradigmas en muchas disciplinas incluyendo a la educación, la cual ha sido mirada últimamente desde otra perspectiva, pues es a través de la Neurodidáctica, que analiza las competencias que el cerebro tiene, que se llega a la comprensión de la diversidad personal en el proceso del aprendizaje, pues aunque las personas cuenten con la misma estructura orgánica, no existen dos que piensen, decidan o actúen de la misma forma.

Finalmente, se puede decir que la diversidad es consecuencia directa de las diferencias y por otro lado, una cualidad del ser humano. Por lo tanto, existe diversidad de estudiantes, diversidad de docentes, diversidad de metodologías educativas, diversidad en la organización de las Instituciones Educativas, diversidad de estrategias para gestionar la diversidad en los centros educativos, pertenecientes a un mundo diverso.

\section{METODOLOGIA}

El artículo presenta los resultados de una investigación desarrollada en el contexto de la Maestría en Neuro pedagogía de la Universidad del Atlántico y está orientado hacia la posibilidad de 
generar un espacio de cualificación para docentes, que a la vez se convierta en escenario para fortalecer la capacidad de reconfigurar su quehacer pedagógico hacía la pertinencia en lo que respecta a la atención a la diversidad presente en los estudiantes, a la luz de los planteamientos de la neurociencia cognitiva y específicamente de la neurodidáctica.

La investigación desarrollada a partir de los planteamientos de la teoría crítica (Guba,1990), desde la cual se movilizan acciones comprometidas con la transformación de la realidad a partir del análisis crítico de las problemáticas del contexto y que llevan a plantear nuevas propuestas.

Desde esta perspectiva, la lógica de causalidad no es lineal sino más bien un entramado de eventos generadores de situaciones susceptibles de ser abordadas y analizadas y en las que de acuerdo con Bonilla y Rodríguez (2000), el investigador se acerca al objeto de estudio para comprender su naturaleza y representarla con el mayor número de elementos posibles, teniendo en cuenta su carácter dinámico y las relaciones entre ellos.

La investigación se desarrolló con 33 docentes de los grados transición y primero de Básica Primaria de las cinco Instituciones Educativas oficiales del municipio de Puerto Colombia, Atlántico, Colombia, distribuidos así:

Tabla 1 Docentes de Transición y Primer grado de las Instituciones Educativas Oficiales del Municipio de Puerto Colombia

\begin{tabular}{lll}
\hline Instituciones Educativas & Docentes de Transición & Docentes de Primero \\
\hline IETT Simón Bolívar & 4 & 3 \\
IE María Mancilla Sánchez & 4 & 4 \\
IE Francisco Javier Cisneros & 5 & 5 \\
IE Eustorgio Salgar & 2 & 2 \\
IE San Nicolás de Tolentino. & 2 & 2 \\
Total & 17 & 16 \\
\hline
\end{tabular}

Fuente: Elaboración propia

A partir de la observación y el establecimiento de relaciones entre teoría y práctica, se logró la transferencia o conexión por parte de los profesores, entre la experiencia vivida en torno a los principios de la neurodidactica y la incorporación de estos a su práctica, específicamente en el tema de la atención a la diversidad. 
El proceso se desarrolló en tres fases, durante la primera, se procedió a plantear la situación problemática a los docentes, generando espacios de análisis de los antecedentes y referentes teóricos que podrían fundamentan las estrategias de atención a la diversidad que los docentes podrían utilizar en transición y primer grado de Básica Primaria.

En el segundo momento, se diseñaron e implementaron talleres, en los que mediante experiencias multisensoriales y lúdicas se trabajó con 33 docentes participantes, el reconocimiento y apropiación de los principios de la neurodidactica, la relación de las funciones ejecutivas con el aprendizaje y la reflexión sobre la acción pedagógica del docente y su capacidad para desarrollar procesos adecuados de atención a la diversidad.

Durante la tercera fase, se realizó un proceso de acompañamiento en el aula, a partir del cual fue posible evidenciar la incidencia de su participación en los talleres. La observación y los testimonios permitieron dar cuenta de una acción pedagógica enriquecida, dinámica y conectada con la perspectiva de atención a la diversidad.

La cuarta fase, estuvo orientada hacia la retroalimentación de la observación con los participantes, para desde allí reiniciar le proceso de planeación de nuevas intervenciones.

\section{HALLAZGOS}

El eje central de la investigación es el reconocimiento por parte de los docentes de Transición y Primer grado de Básica Primaria de los aportes de la neurociencia cognitiva y específicamente de la neurodidactica a la acción pedagógica en el área de atención a la diversidad. En este sentido, fue posible evidenciar en primera instancia que las actividades orientadas a valorar la participación del docente, su experiencia y su voz en la construcción de soluciones a un problema relevante para ellos, es fundamental.

Partiendo de la anterior consideración, fue posible verificar que, si bien los docentes poseen una gran sensibilidad frente a la importancia de desarrollar una acción pedagógica conectada con las necesidades propias de la diversidad de sus estudiantes, muchos no cuentan con los referentes metodológicos o teóricos para hacerlo.

Así, participar y aprender desde la propia experiencia conceptos asociados a la neurodidáctica y su potencial para desarrollar acciones adecuadas de atención a la diversidad en su quehacer docente, se constituyó en un elemento altamente valorado. Durante la observación fue posible verificar la 
planeación de contenidos, teniendo en cuenta las diferencias de los estudiantes, utilizando la lúdica, el juego dramático, partiendo de sus saberes previos, teniendo en cuenta las emociones, la imaginación, la motivación y la cooperación como potenciadores y creadores del conocimiento.

La cualificación de docentes en un tema tan sensible como la atención a la diversidad, conlleva una complejidad inherente a la multiplicidad de factores y elementos que influyen en la deconstrucción y reconstrucción de saberes, teniendo en cuenta que los mismos son producto de un contexto en el que la educación de talla única para todos los estudiantes se imponía como opción en la enseñanza.

A partir de la experiencia vivida, los docentes manifiestan mayor conciencia frente al carácter diverso del contexto donde desarrollan su acción pedagógica y la importancia de enriquecer su práctica a partir de la neuroeducación y específicamente desde la neurodidactica, cuyos principios amplían la mirada del docente frente a las múltiples posibilidades de desarrollar una práctica pedagógica coherente con la diversidad de estilos, ritmos es intereses de sus estudiantes.

Igualmente, se observó que la motivación asociada a participar de una experiencia de cualificación diseñada desde los referentes de la neurodidactica y orientada a ofrecer opciones pertinentes de atención a la diversidad facilito a su vez la creación de otros espacios y experiencias de enseñanza, que evidenciaron el fortalecimiento de un saber hacer en contexto.

La evaluación de la propuesta por parte de los profesores participantes evidenció su consideración frente a la importancia de que si bien, aplicar lo aprendido en los talleres favorece los aprendizajes de los estudiantes, también se ha convertido en un espacio para ampliar su conocimiento, para continuar profundizando en el estudio de la neurodidactica, es decir también generó, una nueva oportunidad de desarrollo profesional.

Otro aspecto importante es la consideración por parte de los docentes, frente a la oportunidad de ampliar la perspectiva respecto de ver en la diversidad propia de los estudiantes una valiosa oportunidad, para desarrollar un quehacer más lúdico, interactivo, multisensorial donde emoción y cognición caminan juntos hacia el logro de objetivos de formación integral de los sujetos.

Además de la motivación intrínseca por participar en los talleres, los docentes reconocen la necesidad de fortalecer la calidad del sistema educativo con propuestas que, desde su voz y experiencia, les permitan reconfigurar su quehacer a partir de las necesidades y potencialidades propias de un contexto diverso, multicultural y pluriétnico en el que se desarrollan cotidianamente su quehacer profesional. 
En este sentido, los profesores afirman que su acción pedagógica ha dado un giro positivo y que el factor más influyente en este cambio es el proceso propiciado por la investigación, pero que debe tener un seguimiento para poder obtener logros duraderos y sostenibles.

\section{DISCUSIÓN}

Al desarrollar esta investigación, se estableció como primer objetivo caracterizar la acción pedagógica de docentes de transición y primer grado del municipio de puerto Colombia en relación con la atención a la diversidad; para verificar el cumplimiento de este objetivo, se recabó información a través de estrategias como la entrevista a los docentes objeto de estudio con las que se facilitó la reflexión y el diálogo. La información arrojada apoya la discusión y análisis de las problemáticas encontradas en el aula a la luz de las teorías, métodos y estrategias neurodidácticas.

El proceso incluyó la entrevista con todos los participantes de forma voluntaria. Cada uno de ellos desarrolló una clase en el curso que lidera desde inicio de año. A partir de esa información se construyeron informes con sus respectivas reflexiones de forma crítica sobre la acción de los docentes, los cuales sirven de insumo para el proceso de triangulación de la información con el fin de caracterizar las prácticas pedagógicas de los docentes participantes.

Con respecto a las observaciones de clase, la acción de los docentes de transición y primer grado del municipio de Puerto Colombia, sus actividades carecen de estrategias efectivas que garanticen la participación de todos los estudiantes sin tener en cuenta la diversidad ni los estilos de aprendizaje. También se evidencia la falta de motivación debida al poco uso de recursos didácticos con actividades monótonas y repetitivas, en donde no se estimulan los saberes previos necesarios para la activación de los saberes ni se planifican con DUA o adecuaciones curriculares, por lo tanto, se puede percibir que la expectativa de aprendizaje es muy baja debido a las clases pasivas desarrolladas de manera tradicional, propias de docentes que no reflexionan que su quehacer.

De la información obtenida en los resultados de la implementación de los instrumentos, se puede realizar el análisis desde las percepciones que tienen los docentes de su acción, en donde ellos reconocen carencias, desconocimientos y desinformación pues no tienen en cuenta la diversidad, debido a que sus clases son planeadas para todos los estudiantes por igual. 
Este estado de la didáctica desarrollada por los docentes objeto de estudio es similar a lo encontrado por Giroux (2000), quien dice que los docentes deben tener el control teórico de todas las maneras existentes de construir la diferencia, pues esta puede presentarse de muchas formas y prácticas. Por lo tanto, es deber del maestro reflexionar en su acción para legitimar esa diferencia y trascender a cualquier frontera cambiante.

Trabajo coherente con el de desarrollado por Gómez (2012), en este trabajo se planteó como objetivo analizar, describir, contrastar e interpretar la gestión de la diversidad y las prácticas directivas inclusivas de los equipos de los centros públicos de Educación Infantil y Primaria de Andalucía acogidos al Plan de Educación Compensatoria. Con el propósito de plantear y diseñar estrategias socioeducativas, curriculares y/o extracurriculares necesarias para posibilitar la integración de los alumnos-as en situación de desventaja sociocultural, estableciendo mecanismos compensadores que contrarresten la influencia de sus condiciones sociales, económicas, familiares y culturales desfavorables (Gómez, 2012, p. 554).

Para dar cumplimiento al segundo objetivo específico que consistió en diseñar una propuesta Neurodidáctica para atención a la diversidad en transición y primer grado de Básica Primaria. Se diseñó para ello la estrategia neurodidáctica N.A.D.A.P Puerto Colombia. (Ver Anexo 4).

Trabajo coherente con el de Tapia (2013), “Diseño y aplicación de un módulo de neurociencias para educadoras de párvulos” trabajo en el que se sometió a un grupo de 16 docentes de preescolar a una estrategia de aprendizaje a través de la implementación del módulo "Neurociencia para el aprendizaje de la Infancia” y por otra parte, se integró un grupo control conformado por 18 docentes de párvulos de los niveles transición I y II en una Escuela Básica de la comuna Estación Central, el cual no participó en la propuesta pedagógica (Tapia, 2013, p. iv). Trabajo relevante para la presente investigación porque realiza aportes importantes desde la práctica docente y la construcción teórica del conocimiento con respecto a la influencia sobre el cerebro y de cómo éste aprende.

De igual manera para dar cumplimiento al tercer objetivo específico relacionado con implementar una propuesta Neurodidáctica para atención a la diversidad en transición y primer grado de Básica Primaria, se implementó para ello la Estrategia N.A.D.A.P Puerto Colombia, la cual estuvo conformada por tres sesiones con actividades para sensibilizar a los docentes en cuanto a cómo la Neurodidáctica y la atención a la diversidad pueden contribuir al mejoramiento de la acción pedagógica de los docentes. 
Trabajo que coincide con el de Madriz, Hidalgo y Antón, (2016), artículo que parte de una investigación abordada desde el modelo de investigación - acción titulada "Desarrollar una propuesta pedagógica, durante el período 2013-2015 para favorecer la estimulación de las competencias comunicativas, dirigida a docentes de preescolar de escuelas de atención prioritaria de las áreas metropolitanas de Costa Rica: Aserrí, Guadalupe y Coronado", como una propuesta de orientada a mejorar la acción pedagógica de los docentes de esas instituciones públicas.

Por último, para dar cumplimiento al cuarto objetivo específico, relacionado con evaluar la incidencia de la propuesta Neurodidáctica para atención a la diversidad en la acción pedagógica de los docentes de transición y primer grado de primaria, se realizó una entrevista a manera de cuestionario virtual.

Según las respuestas de los docentes en cuanto a la aplicación de la estrategia neurodidáctica para la atención a la diversidad N.A.D.A.P Puerto Colombia , se encontró que sintieron motivados debido a la relevancia que estas estrategias presentan para el logro de aprendizajes significativos en los estudiantes, útiles para fortalecer los aprendizajes y por ende el desempeño académico de los estudiantes, pues aportan un alto nivel de conocimiento en las temáticas abordadas a la acción del docente.

De acuerdo a la acción pedagógica de los docentes, haber participado en esta experiencia les inspiró a la reflexión, pues reconocen que sus prácticas no tenían en cuenta la diversidad ni los estilos de aprendizaje, por lo tanto, han empezado a realizar actividades con los estudiantes que tengan significado para ellos, pues tocan sus emociones y permiten el desarrollo cognitivo, comunicativo, motriz y fonológico al mismo tiempo.

En sus respuestas los docentes reconocieron la importancia de tener un buen clima de aula, para poder despertar la motivación, con estrategias que garanticen la interacción de todos los estudiantes, con instrucciones específicas para los diferentes estilos de aprendizaje, de tal manera que el tiempo les alcance para lograr lo propuesto, aprovechando al máximo el uso de recursos y materiales didácticos y la activación de los saberes previos.

Resultados similares con los de Calzadila (2017), que en su artículo "La integración de las neurociencias en la formación inicial de docentes para las carreras de la educación inicial y básica: caso Cuba. El autor afirma en sus conclusiones que es necesario integrar la Neurodidáctica la formación de docentes, pues cada estudiante configura una personalidad única e irrepetible, se fundamenta no solo 
en el carácter biopsicosocial del desarrollo de la personalidad, sino también en el carácter modificable y adaptable de las estructuras cerebrales y de la influencia social.

Por otra parte, estos resultados son coherentes con los de Novoa (2011), quien en su obra "El regreso de los profesores", afirma que el docente debe ser reflexivo y en formación permanente, y este trabajo de formación debe estar próximo a la realidad del escolar, teniendo en cuenta la atención a la diversidad, además de valorizar el trabajo en equipo y el ejercicio colectivo de la profesión (p. 208).

\section{CONCLUSIONES}

El diseño y la aplicación de estrategias neurodidácticas para la atención a la diversidad, tuvo una incidencia positiva en la acción pedagógica de los docentes de transición y primer grado que participaron de ella, pues se familiarizaron con teorías y principios desde los cuales movilizaron actitudes de aceptación y cambio de un nuevo estilo de enseñanza orientado a mejorar las oportunidades de aprendizaje y desarrollo integral de los niños.

La importancia de incorporar la perspectiva neurodidactica a la acción pedagógica del docente enriquece su labor aportándole los elementos necesarios para desarrollar una práctica propositiva, innovadora, creativa, enriquecida y conectada con las necesidades, intereses, ritmos y estilos de aprendizaje de los estudiantes.

Lo anterior teniendo en cuenta que la Atención a la Diversidad es una realidad presente en todas las Instituciones Educativas; por lo tanto, la formación de los docentes debe apuntar al diseño de estrategias neurodidácticas, orientadas a ver en la diversidad propia del contexto educativo, el potencial dinámico, flexible y transformador que debe caracterizar su quehacer.

Un docente, que desde el uso de estrategias neurodidácticas desarrolla un quehacer centrado en la Atención a la Diversidad, tiene la capacidad de reconocer diferentes contextos y ritmos de aprendizaje, identificar recursos que le puedan ser útiles, reflexionar constantemente acerca de su práctica, diseñar e integrar sus actividades evidenciando relaciones e interacciones significativas y experienciales que favorecen a su vez el aprendizaje.

La reflexión constante en torno a la acción pedagógica del docente y su responsabilidad en la atención, respeto y valoración de la diversidad, son elementos inherentes a los procesos de desarrollo profesional, desde los cuales el ser, saber y saber hacer, se movilizan para lograr una sinergia efectiva entre práctica y teoría trascender y la reelaboración del concepto de niño como sujeto diverso. 
En este sentido, se ratifica la importante conexión que debe existir entre lo que el docente aprende, experimenta y conoce en los procesos de desarrollo profesional como el propuesto en la investigación y lo que enseña en el aula, para desde allí enriquecer su labor y favorecer aprendizajes significativos y duraderos en sus estudiantes.

Así, la inclusión en las planeaciones de los docentes de estrategias neurodidacticas, implica necesariamente su participación en procesos continuados de actualización, orientadas a ampliar constantemente las perspectivas desde las cuales logre desplegar en su aula de clase nuevas y creativas formas de aprender a partir de la diversidad y no para la uniformidad.

Estos procesos de cualificación, de acuerdo con la experiencia propiciada por la investigación son posibles en las Instituciones Educativas a partir de la conformación de comunidades de aprendizaje concentradas en el compartir de experiencias, aprendizajes y estrategias a partir de las cuales crear nuevos saberes compartidos.

Lo anterior implica también la movilización de la voluntad institucional para crear tiempos y espacios de formación y de socialización de experiencias, que evidencien la operatividad de políticas específicas de equidad orientadas a transformar la gestión escolar en función de la Atención a la diversidad, lo que facilitará a su vez la posibilidad de trascender del modelo tradicional de enseñanza presente aún en algunas Instituciones Educativas hacia una perspectiva más inclusiva, equitativa y respetuosa de la diversidad.

\section{REFERENCIAS}

Artavia, C., Fallas, M. (2012). Orientación y diversidad: Por una educación valiosa para todos y todas. Revista electrónica Educare. Vol. 16. Pág. 47 - 52. ISSN: 1409-42-58, Costa Rica. Disponible en: https://dialnet.unirioja.es/descarga/articulo/4780997.pdf

Baldwin, P. (2014). El arte dramático aplicado a la educación: Aprendizaje real en mundos imaginarios. Madrid: Morata.

Battro, A., Cardinali, D. (1996). Más cerebro en la educación. La Nación.

Bonilla, E. Rodríguez, P. (2000). Más allá del dilema de los métodos: la investigación en Ciencias sociales. Bogotá, Colombia: Editorial Norma.

Campos, A (2010). Neuroeducación: uniendo las neurociencias y la educación en la búsqueda del desarrollo humano. Revista La Educación. Vol. 143. Disponible en: 
http://www.educoea.org/portal/La_Educacion_Digital/laeducacion_143/articles/neuroeducacion. pdf.

Céspedes, A. (2008b). Cerebro, inteligencia y emoción. edic. Prosa S. A., Chile.

De la Barrera, M., Donolo, D. (2009). Neurociencias y su importancia en contextos de aprendizaje. Revista Digital Universitaria. Vol. 10(4). México. Disponible en: http://www.revista.unam.mx/vol.10/num4/art20/art20.pdf

Dos santos, G. \& Da Silva M. (2020). Educação Inclusiva em uma escola do campo de Teresina (pi): da sala regular ao atendimento educacional especializado (AEE). Cadernos Cajuína, Vol. 5 (3), 194-206104

Giroux, H. (1997). La Pedagogía Crítica. Revista electrónica de educación Sinéctica. Disponible en: https://sinectica.iteso.mx/index.php/SINECTICA/article/view/210/203

Manosalva, S., Tapia, C. (2009). Atender a la diversidad: el control social en la significación de la alteridad (a) normal. Revista de pedagogía crítica Paulo Freire. Vol. 7. Chile. Disponible en: http://revistas.academia.cl/index.php/pfr/article/view/472/612

Montessori, M. (1986). La mente absorbente del niño. México: Diana.

Peralta, M. V. (2005). Nacidos para ser y aprender: La educación en el período más crítico de la vida: los primeros tres años. Buenos Aires: Editorial Infanto Juvenil.

Pineda, A. \& Felicetti, V. (2018). Comprensiones sobre la Practica Pedagógica del profesor, la lúdica en la hora del cuento. Revista Educación. Santa Maria. Vol 43.N. 31.Disponible https://periodicos.ufsm.br/reveducacao/article/view/28323

UNESCO. (1990). Conferencia Mundial de Educación para todos. Recuperado de: https://unesdoc.unesco.org/ark:/48223/pf0000127583_spa

UNESCO. (2000). Foro Mundial sobre la Educación. Dakar, Senegal, 26-28 de abril de 2000: informe final. Recuperado de: https://unesdoc.unesco.org/ark:/48223/pf0000121117_spa

UNESCO. (2008). Conferencia internacional de educación. La educación inclusiva: El camino hacia el futuro. Ginebra. Recuperado de: http://www.ibe.unesco.org/fileadmin/user_upload/Policy_Dialogue/48th_ICE/CONFINTED_4 8-3_Spanish.pdf

UNESCO. (2013). Informe de Seguimiento de la EPT en el mundo. Enseñanza y aprendizaje: Lograr la calidad para todos. Francia. Recuperado de: https://unesdoc.unesco.org/ark:/48223/pf0000225654_spa

UNICEF. (2001). Estado mundial de la infancia. Fondo de las Naciones Unidas para la infancia. Recuperado de: https://www.unicef.org/spanish/sowc01/pdf/fullsowcsp.pdf 
Velásquez, B., Calle, M., Remolina, N., (2006). El cerebro: un mundo de posibilidades para el aprendizaje. Bogotá: Universidad Colegio Mayor de Cundinamarca. 